\title{
Heterogenic colonization patterns by Leptospira interrogans in Rattus norvegicus from urban slums
}

\author{
Ana Amélia Nunes Santos ${ }^{1}$, Cláudio Pereira Figueira ${ }^{2}$, Mitermayer Galvão dos Reis ${ }^{1}$, \\ Federico Costa $^{3,4,5}$, Paula Ristow ${ }^{1,6}$ \\ ${ }^{1}$ Laboratório de Patologia e Biologia Molecular, Fundação Oswaldo Cruz, Salvador, BA, Brazil. \\ ${ }^{2}$ Plataforma de Microscopia Eletrônica, Fundação Oswaldo Cruz, Salvador, BA, Brazil. \\ ${ }^{3}$ Instituto de Saúde Coletiva, Universidade Federal da Bahia, Salvador, BA, Brazil. \\ ${ }^{4}$ Núcleo de Epidemiologia e Bioestatística, Fundação Oswaldo Cruz, Salvador, BA, Brazil. \\ ${ }^{5}$ Division of Epidemiology of Microbial Diseases, Yale School of Public Health, Connecticut, USA. \\ ${ }^{6}$ Laboratório de Bacteriologia e Saúde, Instituto de Biologia, Universidade Federal da Bahia, Salvador, \\ BA, Brazil.
}

Submitted: October 17, 2014; Approved: February 2, 2015.

\begin{abstract}
We evaluated the renal colonization by Leptospira interrogans in Rattus norvegicus (rats), as it is the major natural reservoir of urban leptospirosis. We caught $72 R$. norvegicus, out of which 32 were found to be positive for L. interrogans by immunofluorescence assay. From these rats, we selected 17 and divided them into six groups based on the mass-age/sex. We performed the immunohistochemistry test against $L$. interrogans in the kidney sections of the rats and systematically counted the colonized tubules (CTs) in 20 fields. The proportion of positive fields varied from $5 \%$ to $95 \%$. The number of CTs in 20 fields varied from 0.5 to 85.5 . These differences were not related to age or sex of the animals. The characterization of leptospiral colonization patterns in the natural reservoirs is important to better understand the host-pathogen interactions in leptospirosis.
\end{abstract}

Key words: Leptospira interrogans, renal colonization, natural reservoirs, Rattus norvegicus.

In Brazil, every year around 5,000 patients are reported to have leptospirosis (Brasil Ministério da Saúde, 2012). Weil's syndrome, the severe form of leptospirosis, is caused by pathogenic Leptospira spp., which is characterized by acute renal failure, hepatic failure, and hemorrhages. The mortality rate of this syndrome has reached $15 \%$ (Ko et al., 1999). However, the severe pulmonary hemorrhage syndrome has a mortality rate up to $70 \%$ (Gouveia et al., 2008). The highest prevalence of leptospirosis is reported during heavy rainy seasons in urban settings. In those areas $R$. norvegicus is the major carrier of pathogenic leptospires. They are chronically colonized by these spirochetes in the renal tubules, excreting them in urine, and contaminating the environment (Levett, 2001).

Leptospires colonize the proximal convoluted tubules of $R$. norvegicus, forming dense aggregates of bacteria
(Athanazio et al., 2008; De Faria et al., 2007). Despite cortical bacterial colonization, renal tissue maintains its architecture, presenting slight interstitial nephritis and a small degree of cell degeneration (De Faria et al., 2007). Persistent colonization indicates that kidney is an immune-privileged site that promotes the survival of bacteria (Monahan et al., 2009). Characterization of colonization patterns in chronic reservoirs is important to understand the pathogenic mechanism of Leptospira spp. and its maintenance in the host. However, pathological studies on kidneys of urban rats are scarce. The relationship between the carrier state and demographic factors, such as gender and sex, or environmental predisposing factors, has not yet been studied in synanthropic rats. We aimed to characterize renal colonization by $L$. interrogans in $R$. norvegicus of different age and sex caught in slum areas with high incidence of severe leptospirosis in Salvador, Bahia, Brazil. 
This study was performed in Pau da Lima and Sete de Abril, two neighborhoods in Salvador, Bahia, Brazil, with high incidence of severe leptospirosis (Reis et al., 2008). The rats were systematically caught from June to September 2010. The sampling methodology involved setting of 20-25 Tomahawk traps in peridomiciliary areas of five continuous households at each site (seven sites were located in Pau da Lima and two sites in Sete de Abril), for 5 days (Porter et al., 2015). The households had a previous history of severe human leptospirosis, and they were geo-referenced and selected randomly (Reis et al., 2008). The caught rats were then transported to the Zoonosis Control Center, where they were euthanized, and the kidneys were collected during necropsies that followed biosafety protocol levels 2 and 3 (Mills et al., 1995).

As a qualitative method, the immunofluorescence (IF) imprint technique was carried out to screen the kidneys for $L$. interrogans infection, following the exact protocol described by Chagas-Junior et al. (2012). Kidneys were collected and preserved in $10 \%$ buffered formalin. Data were collected on standardized questionnaires, entered, and validated in a unique database (Epi Info system). Rats positive for IF were classified into six groups according to age and sex: juvenile males $(\leq 200 \mathrm{~g})$, juvenile females $(\leq 200 \mathrm{~g})$, young adult males (201-399 g), young adult females (201-399 g), adult males ( $\geq 400 \mathrm{~g}$ ), and adult females ( $\geq 400 \mathrm{~g}$ ). For each group, three rats were randomly selected: two IF positive and one IF negative as a negative control.

Formalized kidneys were embedded in paraffin wax, and then $2-\mu \mathrm{m}$ sections were stained with hematoxylineosin (HE) and submitted to immunohistochemistry (IH) anti-L. interrogans for histopathology and leptospiral colonization studies, respectively. For the IH test, we followed the protocol described by Croda et al. (2008), with some modifications, which are as follows: tissues were deparaffinized, sections were treated with $0.3 \%$ hydrogen peroxide for $15 \mathrm{~min}$ at room temperature (RT), blocked by incubation with $10 \%$ skimmed milk in $1 \mathrm{x}$ Phosphate Buffered Saline (PBS), and incubated with primary antibodies (1,000-fold dilution of polyclonal rabbit antibody anti- $L$. interrogans serovar Icterohaemorrhagiae strain RGA) at RT for $1 \mathrm{~h}$. Samples were washed with $1 \%$ PBS and then incubated at RT for 20 min with secondary antibodies from the Histostain-Plus kit (Invitrogen), adding 5\% of nonimmune rat serum to avoid unspecific reactions. Enzyme reactions were tested using 3,3'-diaminobenzidine (Sigma). Microscopic observations were performed using an Olympus BX51 optical microscope. Two independent and blinded observers performed screening observations of IH sections. Rats positive for the IF test, but negative for the IH test, were excluded from this study.

To evaluate the distribution and intensity of renal colonization by IH, each renal section was divided into quadrants. For each quadrant, we randomly selected five fields in the cortex, totaling 20 fields. Observations were performed using 40x objective with two readings per slide. The number of colonized tubules (CTs), positively marked by IH anti- $L$. interrogans, was registered for all fields (CT 20 count). The CT 20 count was considered a quantitative measurement of infection. The arithmetic mean between the two readings for the number of CT 20 count and the number of CTs per field was obtained. Descriptive analysis was performed to compare the quadrants and field positivity, as well as the number of positive CTs between sex and mass-age groups. The Mann-Whitney test (Epi Info) was performed to compare the differences in $C T 20$ count related to sex and age.

This work was approved by the Institutional Animal Care and Use Committee at the Oswaldo Cruz Foundation (Salvador, Brazil; protocol number 003/2012).

We caught 72 rats (R. norvegicus), out of which 32 were found to be positive for $L$. interrogans by IF assay $(44 \%)$, and the kidneys were preserved in formalin for posterior IH analysis. We randomly selected 11 positive rats and 6 negative rats and divided them into six groups based on the mass-age/sex. In one group (juvenile males), it was not possible to come across two positive rats $(n=1)$. The six IF-negative control rats were confirmed to have a negative $\mathrm{IH}$ result.

In the kidney $\mathrm{IH}$ analysis using anti- $L$. interrogans antibodies, it was found that the proportion of positive cortical quadrants varied from $25 \%$ to $100 \%$, with high variation between the groups (Table 1). The proportion of fields containing positive CTs was also heterogenic and varied from $5 \%$ to $95 \%$, with the maximum percentage obtained in one juvenile female and one adult male. Also, there was a wide variation in the total $C T 20$ count, with a minimum value of 0.5 and a maximum of 85.5 , demonstrating large heterogeneity in the infection patterns in the carrier rats (Table 1). The highest values of CT 20 count (83.5 in an adult male and 85.5 in a juvenile female) were observed in two rats, corresponding to $18.2 \%$ of the sample number. Other animals presented much lower CT 20 count values, from 0.5 to 26.5 (Table 1).

The proportion of positive rats (44\%) was smallest compared with those previously reported in the literature. Recently, Costa et al. (2014) studied 84 rats and found the prevalence of infection by $L$. interrogans to be $63.1 \%$. In a previous study, De Faria et al. (2007) studied 60 rats and found $82 \%$ of prevalence. For all criteria examined regarding the colonization patterns in the IH test, we observed differences in colonization between individuals, regardless of the group to which they belonged. However, no significant differences were observed in kidney colonization (CT 20 count $)$ of rats between sex $(\mathrm{p}=0.33)$ or mass-age groups $(\mathrm{p}=0.67)$. These findings may be related to other individual characteristics or to environmental factors, such as the bacterial inoculum in the environment. Although we did not assess the environmental bacterial contamination, the 
Table 1 - Proportions of $R$. norvegicus kidneys colonized by L. interrogans and number of colonized tubules (CT) by immunohistochemistry.

\begin{tabular}{|c|c|c|c|c|}
\hline & CT 20 count $^{* 1}$ & $\%$ of quadrants with $\mathrm{CT}^{* 2}$ & $\%$ of fields with $\mathrm{CT}^{* 3}$ & Mean no. of CT per field \\
\hline \multicolumn{5}{|l|}{ Females } \\
\hline Juvenile 1 & 85.5 & 100 & 95 & 4.275 \\
\hline Juvenile 2 & 7 & 100 & 40 & 0.35 \\
\hline Young adult 1 & 19 & 75 & 40 & 0.95 \\
\hline Young adult 2 & 26.5 & 100 & 55 & 0.875 \\
\hline Adult 1 & 25 & 100 & 55 & 1.25 \\
\hline Adult 2 & 0.5 & 25 & 5 & 0.025 \\
\hline \multicolumn{5}{|l|}{ Males } \\
\hline Juvenile & 2.5 & 50 & 10 & 0.125 \\
\hline Young adult 1 & 17 & 100 & 45 & 0.85 \\
\hline Young adult 2 & 10 & 50 & 15 & 0.5 \\
\hline Adult 1 & 5.5 & 25 & 5 & 0.275 \\
\hline Adult 2 & 83.5 & 100 & 95 & 4.175 \\
\hline
\end{tabular}

${ }^{*}$ Obtained by the mean of two counts of CT number in 20 randomly selected fields. ${ }^{{ }^{2}} \%$ of quadrants containing CT. ${ }^{*_{3}} \%$ of fields containing CT in 20 randomly selected fields.

study site is endemic to leptospirosis, based on wide active surveillance for severe cases (Reis et al., 2008). It was already shown that the presence of open sewers or garbage accumulation in peridomiciliary areas influences the risk of human infection with Leptospira spp. (Reis et al., 2008). These factors could also be important for the risk of leptospiral infection in rats, and at the end, determine the individual differences in colonization. Another justification for the differences found in our study could be individual responses to the presence of the parasite and/or specific immune response. The lack of significant differences in this study regarding sex and mass-age may also be related to the small sample number, which is a limitation in this study.

The patterns of colonization, according to the distribution of positive CTs in the cortex, varied among the 11 IH-positive rats: three exhibited widespread colonization throughout the cortex, while eight showed agglomeration of CTs in different cortical regions (Figure 1). Slight differences could be observed according to the intensity of IH staining of the tubules. Half of the rats had a pattern of staining restricted to the membrane of renal epithelium. The other half also had a pattern of staining in the whole lumen of the tubule (complete occlusion of the CT; Figure 1), in agreement with what was previously shown by Athanazio et al. (2008). However, there is no classification in the literature about the different patterns of renal colonization in rats or in other reservoirs of leptospirosis. These findings may be related to particularities of each individual regarding the transmission route, bacterial load, immune response, or environmental factors that influence the infection.

HE revealed preservation of renal tissue architecture. A small degree of cell degeneration was evidenced by the presence of tubular amorphous pink material. Other find-

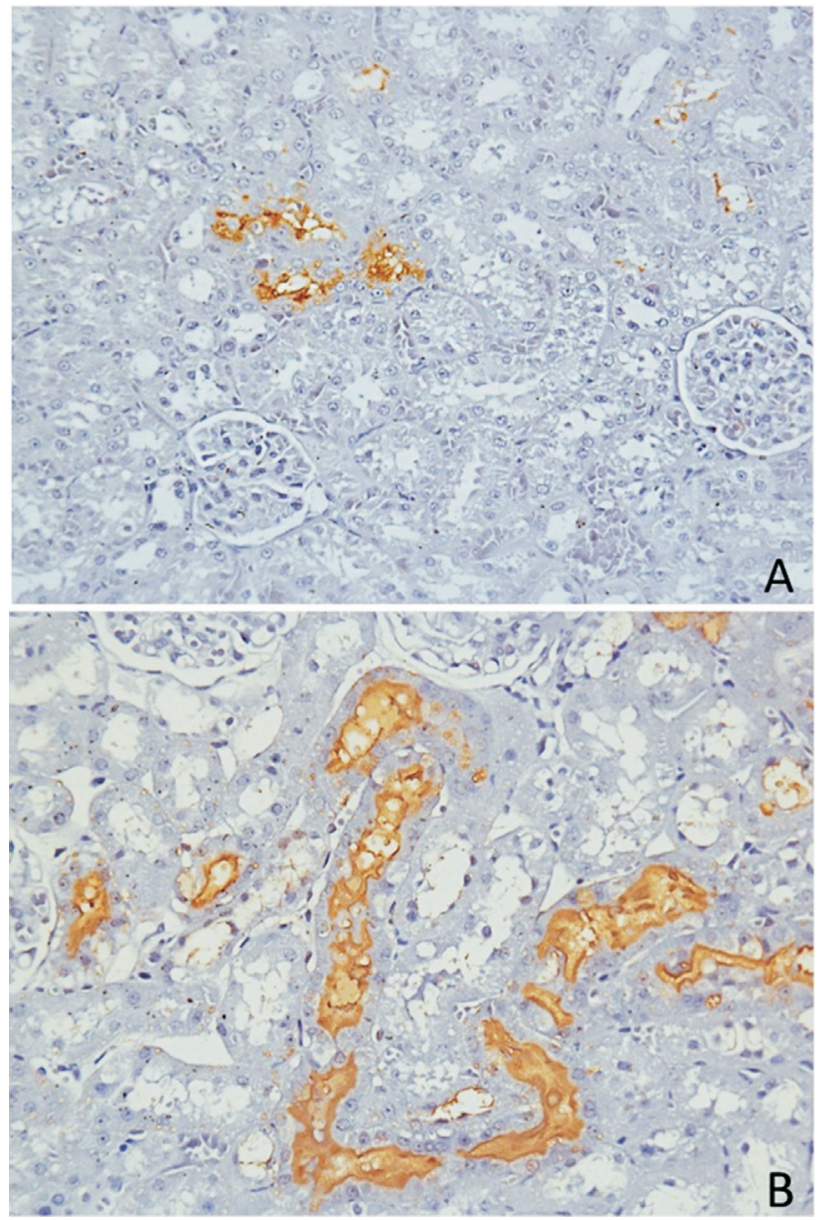

Figure 1 - Kidney sections of $R$. norvegicus stained positively for immunohistochemistry (IH) anti-L. interrogans. In A, we observed a low $\mathrm{CT}$ count and the tubules presented with IH staining restricted to the membrane of epithelial renal cells. In $\mathbf{B}$, massive colonization is shown, with a high CT count, and the tubules also presented a pattern of $\mathrm{IH}$ staining occluding the lumen. Magnification: 400x. 
ings were the presence of a small degree of hyaline droplet degeneration and a minimal amount of inflammatory infiltrate, agreeing with renal pathology previously described by De Faria et al. (2007).

We have characterized the colonization aspects in the kidneys of rat reservoirs of leptospirosis. The heterogeneity of colonization patterns, regardless of age or sex, suggests the possible influence of other host or environmental factors affecting animal maintenance of leptospirosis. This study will contribute to the better understanding of the pathology and pathogenesis of $L$. interrogans in host reservoirs.

\section{Acknowledgments}

We thank Prof. Albert Ko, Dr. James Childs and Fleur Porter for study design; Dr. Daniel Athanazio and Dr. Eduardo Ramos for assistance with histopathology; the staff of the Zoonosis Control Center of Salvador and the Service of Histotechnology of CPqGM/FIOCRUZ for technical assistance. This work was supported by Oswaldo Cruz Foundation (FIOCRUZ), the Secretariat of Health Surveillance of the State of Bahia, the National Institutes of Health (grants: R01 AI052473, R01 TW009504, R25 TW009338), and the Wellcome Trust (102330/Z/13/Z).

\section{References}

Athanazio DA, Silva EF, Santos CS et al. (2008) Rattus norvegicus as a model for persistent renal colonization by pathogenic Leptospira interrogans. Acta Trop 105:176-180.

Brasil Ministério da Saúde (2012) Indicadores e Dados Básicos para a Saúde - Brasil. Ministério da Saúde, Brasília.

Chagas-Junior AD, da Silva CLR, Soares LM et al. (2012) Detection and Quantification of Leptospira interrogans in Ham- ster and Rat Kidney Samples: Immunofluorescent Imprints vs. Real-time PCR. PLoS One 7:e32712.

Costa F, Porter F, Rodrigues G et al. (2014) Infections by Leptospira interrogans, Seoul virus and Bartonella spp. among Norway Rats (Rattus norvegicus). Vector Borne Zoonotic Dis 14:33-40.

Croda J, Figueira CP, Wunder EAJr et al. (2008) Targeted mutagenesis in pathogenic Leptospira species: disruption of the $\operatorname{Lig} B$ gene does not affect virulence in animal models of leptospirosis. Infect Immun 76:5826-5833.

De Faria MT, Athanazio DA, Ramos EAG et al. (2007) Morphological alterations in the kidney of rats with natural and experimental Leptospira infection. J Comp Pathol 137:231-238.

Gouveia E, Metcalfe J, de Carvalho AL et al. (2008) Leptospirosis-associated severe pulmonary hemorrhagic syndrome, Salvador, Brazil. Emerg Infect Dis 14:505-508.

Ko AI, Reis MG, Dourado CMR et al. (1999) Urban epidemic of severe leptospirosis in Brazil. Lancet 354:820-824.

Levett PN (2001) Leptospirosis. Clin Microbiol Rev 14:296-326.

Mills JN, Childs JE, Ksiazek TG et al. (1995) Methods for Trapping and Sampling Small Mammals for Virologic Sesting. Services USDoHaH Ed. Atlanta.

Monahan AM, Callanan JJ, Nally JE (2009) Review Paper: HostPathogen Interactions in the Kidney during Chronic Leptospirosis. Vet Pathol 46:792-799.

Porter FH, Costa F, Rodrigue G et al. (2015) Morphometric and demographic differences between tropical and temperate Norway rats (Rattus norvegicus). J Mammal 96:317-323.

Reis RB, Ribeiro GS, Felzemburgh RDM et al. (2008) Impact of Environment and Social Gradient on Leptospira Infection in Urban Slums. PLoS Negl Trop Dis 2:e228.

Associate Editor: Marina Baquerizo

All the content of the journal, except where otherwise noted, is licensed under a Creative Commons License CC BY-NC. 